\title{
TWO HUNDRED METRE PENDULUM
}

\author{
T. J. КUKКАMÄKI
}

\author{
Finnish Geodetic Institute
}

In measuring the absolute gravity by the aid of any type of pendulum it has always been difficult to determine the actual point of suspension. The effect of the errors occurring here, as well as errors caused by the deformations in suspending parts, is possible to make smaller by using longer pendulums than hitherto.

In the Finnish Geodetic Institute the following arrangement for measurement of the absolute gravity has been planned and some preparatory experiments to this aim have been carried out.

A pendulum of length $220 \mathrm{~m}$ is to be suspended in a mine shaft of $230 \mathrm{~m}$ depth. The head of pendulum a $20 \mathrm{~kg}$ spherical body of brass or of some other material is hanging on a line as light as possible. The pendulum is suspended alternating from the top end and from the middle of the line, and the gravity is evaluated from the difference of these two pendulum lengths and so the most part of errors in determination of suspending points and of gravity centre can be eliminated. The difference of the lengths of pendulums is measured by the air of Väisälä light-interference comparator and then an accuracy of $0.01 \mathrm{~mm}$, i.e. one to $10 \mathrm{milj}$ is to be attained. Changes in the pendulum length during the observation are controlled by optical microscope observation and there differential accuracy of $0.01 \mathrm{~mm}$ is attainable. The oscillation times are observed by the aid of a photocell and a quartz clock as then an accuracy of $0.0001 \mathrm{sec}$ in one passage of pendulum is possible and so for example the measurement of 100 oscillations, i.e. a 30 min observation interval gives an accuracy of one to 10 milj.

The effect of the surrounding air will be a difficult problem, which we try to solve by using different pendulum bodies and lines of the same outside dimensions but of different weights. If this will not give the expected accuracy a vertical tube will be erected and evacuated satisfactorily.

It seems to be possible to attain an accuracy of one to 10 milj in all different partial steps of the measurement and so it is expected that the total error will remain below one to milj, i.e. below $1 \mathrm{mgal}$. 